\title{
Dark energy equation of state parameter and its evolution at low redshift
}

\author{
Ashutosh Tripathi ${ }^{a, b}$ Archana Sangwan $^{a}$ H. K. Jassal ${ }^{a, 1}$ \\ ${ }^{a}$ Indian Institute of Science Education and Research Mohali, \\ SAS Nagar, Mohali 140306, Punjab, India. \\ ${ }^{b}$ Center for Field Theory and Particle Physics and Department of Physics, \\ Fudan University, 200433 Shanghai, China. \\ E-mail: ashutosh_tripathi@fudan.edu.cn, archanakumari@iisermohali.ac.in, \\ hkjassal@iisermohali.ac.in
}

\begin{abstract}
In this paper, we constrain dark energy models using a compendium of observations at low redshifts. We consider the dark energy as a barotropic fluid, with the equation of state a constant as well the case where dark energy equation of state is a function of time. The observations considered here are Supernova Type Ia data, Baryon Acoustic Oscillation data and Hubble parameter measurements. We compare constraints obtained from these data and also do a combined analysis. The combined observational constraints put strong limits on variation of dark energy energy density with redshift. For varying dark energy models, the range of parameters preferred by the supernova type Ia data is in tension with the other low redshift distance measurements.
\end{abstract}

\footnotetext{
${ }^{1}$ Corresponding author.
} 


\section{Contents}

1 Introduction 1

2 Cosmological Equations $\quad 2$

3 Observations 3

4 Analysis and Results 5

5 Summary and Conclusions $\quad 8$

\section{Introduction}

The acceleration of the cosmic expansion is one of the most important discoveries in present day cosmology [1-9]. This acceleration requires that nearly three-quarters of the energy of the universe is in a component with a negative pressure, namely dark energy. A large number of models have been proposed in an attempt to explain dark energy. The simplest of these models is the cosmological constant models and this model is consistent with observations. However, due to the fine tuning problem [11-22] models of dark energy have been proposed; based on fluids and on canonical and noncanonical scalar fields [23-26]. The condition for late time acceleration is that the equation of state parameter of dark energy is $w<-1 / 3$, where $w$ is the ratio of pressure $p$ and the energy density $\rho$. The equation of state parameter can be a constant or it can be a function of time.

A simple model of dark energy with a constant or a varying equation of state parameter is that of a barotropic fluid. A parameterized form of $\mathrm{w}(\mathrm{z})$ is assumed for varying dark energy. The parameterization may, for example, be a Taylor series expansion in the redshift, a Taylor series expansion in the scale factor or any other general parameterization for $w(z)$ [27-42]. The parameters can then be constrained using different observations. For detailed reviews, see [43-45].

In this paper we study dark energy modeled by a barotropic fluid, in the context of present observations. We consider three different scenarios, namely, a constant equation of state parameter of dark energy, and three different parameterizations of dark energy parameter with a variable $w$. In this paper, we use type Ia supernova (SNIa) data [1-9], Baryon Acoustic Oscillation(BAO) data [46-52] and Hubble parameter $(\mathrm{H}(\mathrm{z}))$ data [53-62]. Other potential observational constraints include those from Gamma Ray Bursts (GRB) data [63-67], angular size versus redshift data [68, 69], Cosmic Microwave Background (CMB) data [70, 71]. The GRB and angular size versus redshift datasets, as yet, are not as restrictive as SNIa, BAO, $\mathrm{H}(\mathrm{z})$ or $\mathrm{CMB}[70,71]$. In this paper, we concentrate on dark energy equation of state parameter value and its evolution at low redshifts. Since we are investigating the low redshift behavior of dark energy, we consider the SNIa, BAO and $\mathrm{H}(\mathrm{z})$ data for this analysis. In particular, we investigate if one functional form of the dark energy parameters is preferred over the others by observations. We demonstrate that combined analysis from these datasets constrain the allowed range of parameters significantly. The constraints obtained from observations are consistent with a cosmological constant.

The paper is structured as follows. After introductory Section 1, in section 2, we review background cosmology and in section 3, different observations are discussed and used to constrain different models. Section 4 presents our results and the last section 5 summarizes the main results and concludes. 

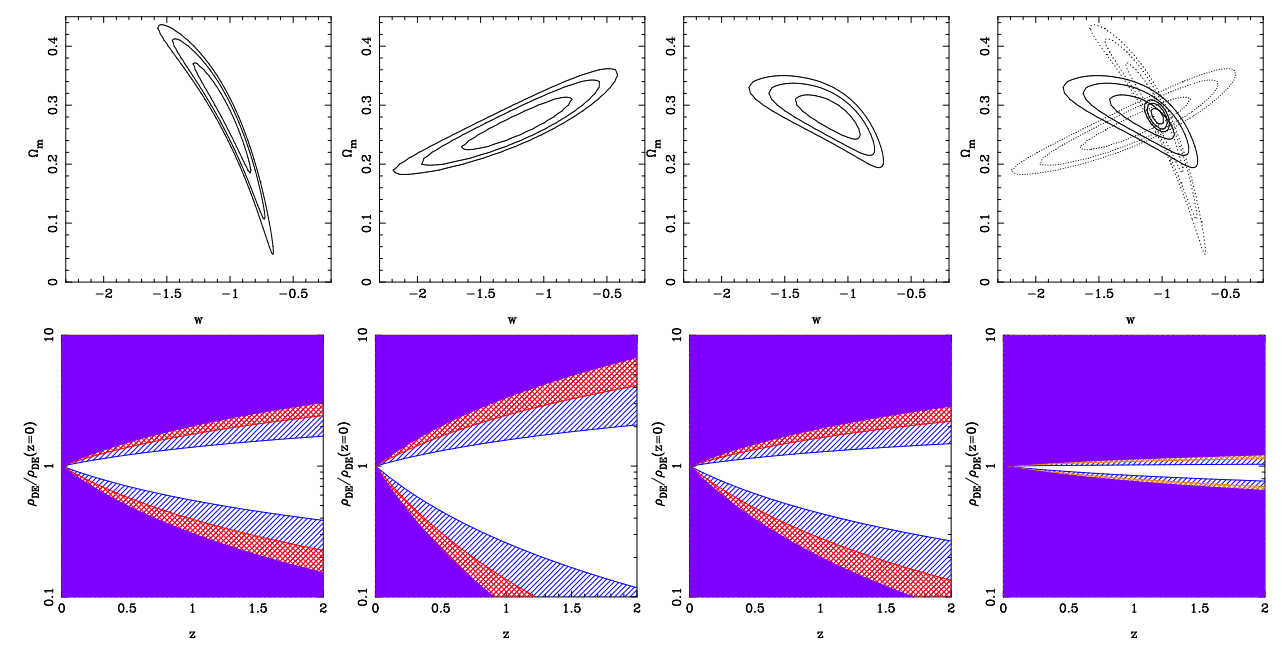

Figure 1. The first row of the figure represents confidence contours at $1 \sigma, 2 \sigma$ and $3 \sigma$ in $\Omega_{m}-w$ plane. Going from left to right, the results are for SNIa, BAO and $\mathrm{H}(\mathrm{z})$ data respectively. The fourth plot represents the combined result. The plots in the second row represents dark energy density versus redshift for the aforementioned data sets and their combination in the same order as before. The white region in the middle is the allowed range of dark energy density at $1 \sigma$ level and the regions with slanted lines (Blue) and hatched lines (red) allowed $2 \sigma$ and $3 \sigma$ ranges respectively. The solid blue region is ruled out.

\section{Cosmological Equations}

A homogeneous and isotropic universe is described by Friedmann equations which for a spatially flat geometry are given by

$$
\begin{aligned}
\frac{\dot{a}^{2}}{a^{2}} & =\frac{8 \pi G \rho}{3} \\
\frac{2 \ddot{a}}{a}+\frac{\dot{a}^{2}}{a^{2}} & =-8 \pi G P
\end{aligned}
$$

where $\rho$ is the total energy density and $P$ is the pressure of the universe[73]. We have assumed speed of light $c$ to be unity. The total energy density is given by

$$
\rho=\rho_{m}+\rho_{R}+\rho_{D E}
$$

where the subscripts $m, R$ and $D E$ denote the non-relativistic, relativistic (including radiation) and the dark energy components respectively. The non-relativistic matter includes baryonic matter and dark matter. The total energy density can also be expressed in terms of density parameters $\Omega_{i}=$ $\rho_{i} / \rho_{c}$, where $i$ indicates the values of different components of $\rho$ given in Eq. 2.2 and $\rho_{c}$ is the critical energy density of the universe, which is given by $\rho_{c}=3 H_{0}{ }^{2} / 8 \pi G$. Since the relativistic component is subdominant at late times, for a spatially flat universe $\Omega_{m}+\Omega_{D E} \approx 1$. In other words, the energy density of the universe comprises primarily of the nonrelativistic matter and dark energy components. Therefore, the first equation in Eq. 2.1 can be rewritten as

$$
H^{2}=H_{0}^{2}\left[\Omega_{m}(1+z)^{3}+\Omega_{D E} \exp \left\{3 \int_{0}^{z} \frac{d z}{1+z}[1+w(z)]\right\}\right]
$$

where $w$ is the equation of state parameter for dark energy component and $z$ is the redshift. 
We consider two different models; one with a constant $w$ and another with a varying $w$. The varying equation of state parameter can be approximated by a function of redshift. Although many parameterizations have been proposed, we use the following parametrizations in this analysis:

$$
w(z)=w_{0}+w^{\prime}(z=0) \frac{z}{(1+z)^{p}} ; \quad p=1,2
$$

and the logarithmic paramterization, given by

$$
w(z)=w_{0}+w^{\prime}(z=0) \log (1+z)
$$

where $w_{0}$ is the value of equation of state parameter at present and $w^{\prime}(z=0)$ is the first order derivative of $w(z)$ at $z=0$. The redshift behavior is different in these two parameterizations. If $p=1[27,28]$, the asymptotic value of $\mathrm{w}(\mathrm{z})$ at high redshifts is $w(z=\infty)=w_{0}+w^{\prime}$ and for the parameterization $p=$ $2, w(z=\infty)=w_{0}$ [29]. The equation of state parameter increases monotonically for the logarithmic parameterization [30]. The present value of the equation of state parameter is $w(0)=w_{0}$ for all these parameterizations.

The evolution of dark energy density $\left(\rho_{D E}\right)$ with the expansion of the universe for these equation of state parameters are then given by

$$
\begin{gathered}
\frac{\rho_{D E}}{\rho_{D E_{0}}}=(1+z)^{3\left(1+w_{0}+w^{\prime}\right)} \exp \left[-\frac{3 w^{\prime} z}{1+z}\right] \\
\frac{\rho_{D E}}{\rho_{D E_{0}}}=(1+z)^{3\left(1+w_{0}\right)} \exp \left[\frac{3 w^{\prime}}{2}\left(\frac{z}{1+z}\right)^{2}\right]
\end{gathered}
$$

and

$$
\frac{\rho_{D E}}{\rho_{D E_{0}}}=(1+z)^{3\left(1+w_{0}\right)} \exp \left[\frac{3 w^{\prime}(z=0)}{2}(\log (1+z))^{2}\right]
$$

for $p=1$ and $p=2$ and logarithmic parameterisation respectively. Here, $\rho_{D E_{0}} \equiv \rho_{D E}(z=0)$, which is the present value of dark energy density, and $w^{\prime} \equiv d w / d z$ at the present time.

\section{Observations}

The three data sets we have used in this analysis are the supernovae (SNIa) observations, Hubble parameter(H(z) observations and the baryon acoustic oscillation(BAO) data. Type Ia supernovae are standard candles and are useful in determining the expansion history of the universe [1-9]. The supernova's apparent brightness determines its distance from the observer and the time taken by photons to reach to the observer. By comparing the flux to redshift relation for numerous SNIa, the rate of expansion of universe and its variation with time can be determined. The relation of luminosity distance to redshift $z$ is given by

$$
d_{L}(z)=\frac{c}{H_{0}}(1+z) \int_{0}^{z} d_{H}(z) d z
$$

where $d_{H}$ is the Hubble radius. For a spatially flat universe, and if contribution of radiation is ignored, $\Omega_{m}+\Omega_{D E}=1$. In this case, $d_{H}$ is given by

$$
d_{H}(z)=\left[\Omega_{m}(1+z)^{3}+\Omega_{D E} \exp \left\{3 \int_{0}^{z} \frac{d z}{1+z}[1+w(z)]\right\}\right]^{-1 / 2} .
$$




\begin{tabular}{|c|c|c|}
\hline Parameter & Lower Limit & Upper Limit \\
\hline$\Omega_{m}$ & 0.01 & 0.6 \\
\hline $\mathrm{W}$ & -4.0 & 0.0 \\
\hline$H_{0}$ & 65.0 & 75.0 \\
\hline
\end{tabular}

Table 1. This table lists the priors used for parameter fitting in case of $w \mathrm{CDM}$ model.

\begin{tabular}{|c|c|c|}
\hline Parameter & Lower Limit & Upper Limit \\
\hline$\Omega_{m}$ & 0.1 & 0.6 \\
\hline$w_{0}$ & -5.0 & 2.0 \\
\hline$w^{\prime}(z=0)$ & -10.0 & 10.0 \\
\hline$H_{0}$ & 65.0 & 75.0 \\
\hline
\end{tabular}

Table 2. This table lists the priors used in the parameter fitting, when $w$ is a function of redshift.

For a $\Lambda C D M$ model the equation of state is $w=-1$. The equation can be further written as

$$
d_{H}(z)=\left[\Omega_{m}(1+z)^{3}+\Omega_{D E}\right]^{-1 / 2} .
$$

For a $w$ CDM model, $w \neq-1$ and is a constant. The Eq. 3.2 then modifies as

$$
d_{H}(z)=\left[\Omega_{m}(1+z)^{3}+\Omega_{D E}(1+z)^{3(1+w)}\right]^{-1 / 2} .
$$

The SNIa data comprises of distance modulus at a given redshift along with the associated error [9]. The distance modulus, $\mu$, is defined as

$$
\mu=5 \log \left(d_{L}\right)-5
$$

in $10 p c$ units.

The second dataset we have used is that of Baryon Acoustic Oscillations [46-52]. Before the recombination epoch, baryons are tightly coupled to photons via the Thompson scattering. The competition between the pressure and gravity leads to acoustic oscillations. These sound waves are imprinted in the baryon perturbations, and give rise to baryon acoustic oscillation peaks. These constitute an independent probe of dark energy as they provide a standard ruler for length scale in cosmology, namely the radius of the sound horizon. The effective distance ratio is defined as

$$
D_{v}(z)=\left[\frac{(1+z)^{2} d_{A}^{2}(z) c z}{H(z)}\right]^{1 / 3},
$$

where $d_{A}$ is the angular diameter distance. Another description has been introduced by Eisenstein [46], where the acoustic parameter is defined as

$$
A(z)=\frac{100 D_{v}(z) \sqrt{\left(\Omega_{m} h^{2}\right)}}{c z} .
$$

The BAO data comprises of $A(z)$ and $d_{z}(z)$ along with errors associated with them for different values of $z$ [46-52]. For our analysis, we have used the Acoustic Parameter for six data points from [50] and the parameter $D_{V}(z)$ for the three data points in $[51,52]$.

The value of Hubble parameter at different redshifts is also a useful tool to constrain the cosmological parameters [53-62]. The Hubble parameter $\mathrm{H}(\mathrm{z})$ is measured using different techniques. The data comprises of 28 independent measurements of $\mathrm{H}(\mathrm{z})$, listed in references in [53]. 

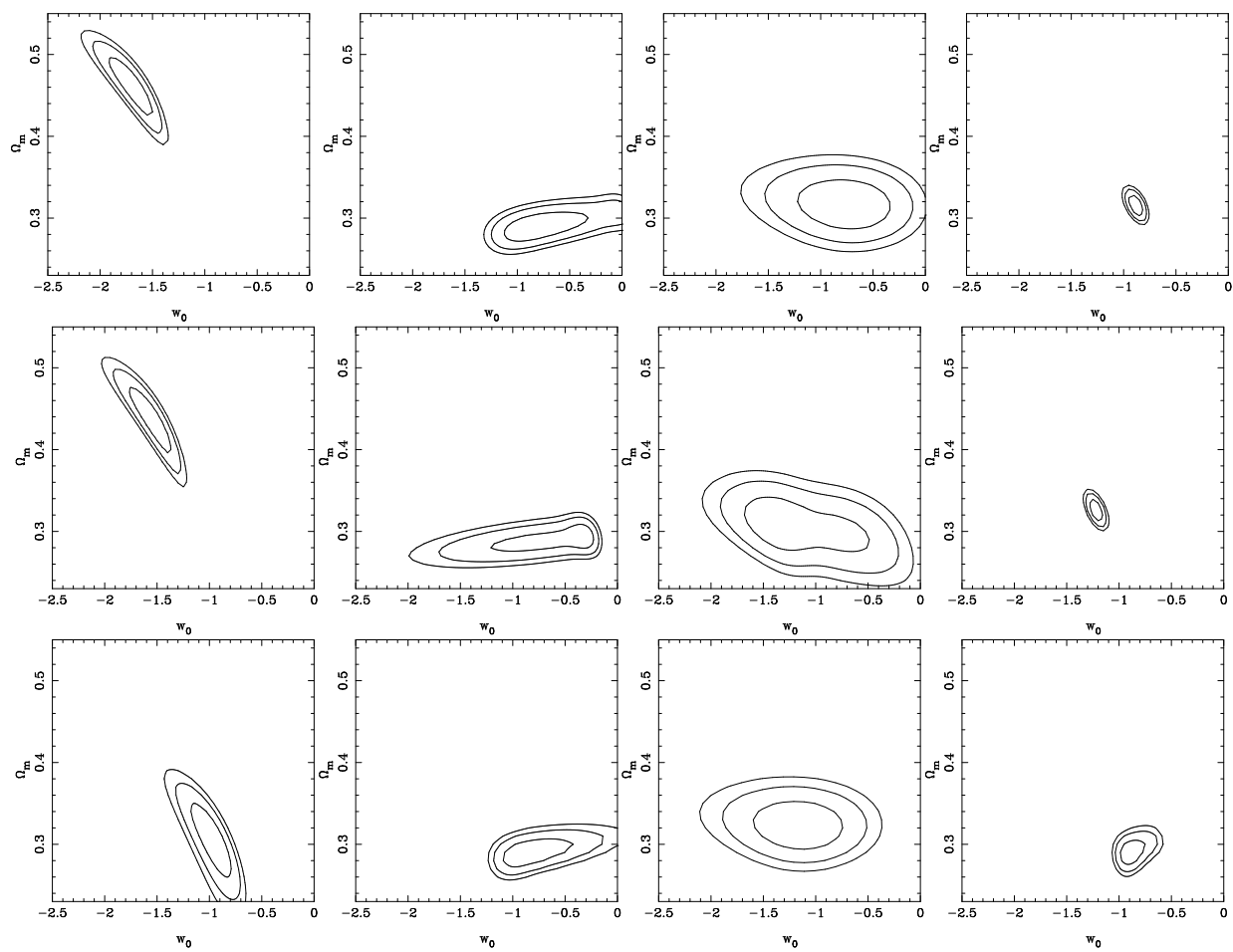

Figure 2. The plots represent the confidence contours for (from left to right) SNIa, BAO, H(z) and a combination of the datasets for the two different parameterizations with marginalization over $w^{\prime}$. The contours in first and second row are obtained for parameterizations $p=1$ and $p=2$ respectively and plots in the last row are for the logarithmic paramterization.

\begin{tabular}{|l|l|l|l|}
\hline Data set & $3 \sigma$ confidence & $\chi_{m}^{2}$ & Best Fit Model \\
\hline SNIa & $0.05 \leq \Omega_{m} \leq 0.43$ & 562.29 & $\Omega_{m}=0.29$ \\
& $-1.57 \leq \mathrm{w} \leq-0.66$ & & $\mathrm{w}=-1.04$ \\
& & & \\
\hline BAO & $0.19 \leq \Omega_{m} \leq 0.36$ & 0.96 & $\Omega_{m}=0.27$ \\
& $-2.19 \leq \mathrm{w} \leq-0.42$ & & $\mathrm{w}=-1.17$ \\
& & & \\
\hline $\mathrm{H}(\mathrm{z})$ & $0.2 \leq \Omega_{m} \leq 0.35$ & 16.27 & $\Omega_{m}=0.28$ \\
& $-1.78 \leq \mathrm{w} \leq-0.72$ & & $\mathrm{w}=-1.12$ \\
& & & \\
\hline SNIa+BAO+H(z) & $0.25 \leq \Omega_{m} \leq 0.31$ & 580.81 & $\begin{array}{l}\Omega_{m}=0.28 \\
\mathrm{w}=-1.03\end{array}$ \\
& $-1.13 \leq \mathrm{w} \leq-0.95$ & & \\
\hline
\end{tabular}

Table 3. This table shows the $3 \sigma$ confidence limit for three datasets, for cosmological models where dark energy parameter is a constant ( $w C D M$ cosmology).

\section{Analysis and Results}

The SNIa UNION compilation data [9] comprises of distance moduli of 580 supernovae upto $z \sim 1.4$. The BAO observations are compiled from a variety of observations [46-52] and cover redshift up to $z=2.3$. The third data we use are estimates of the Hubble parameter $\mathrm{H}(\mathrm{z})$ at different redshifts 

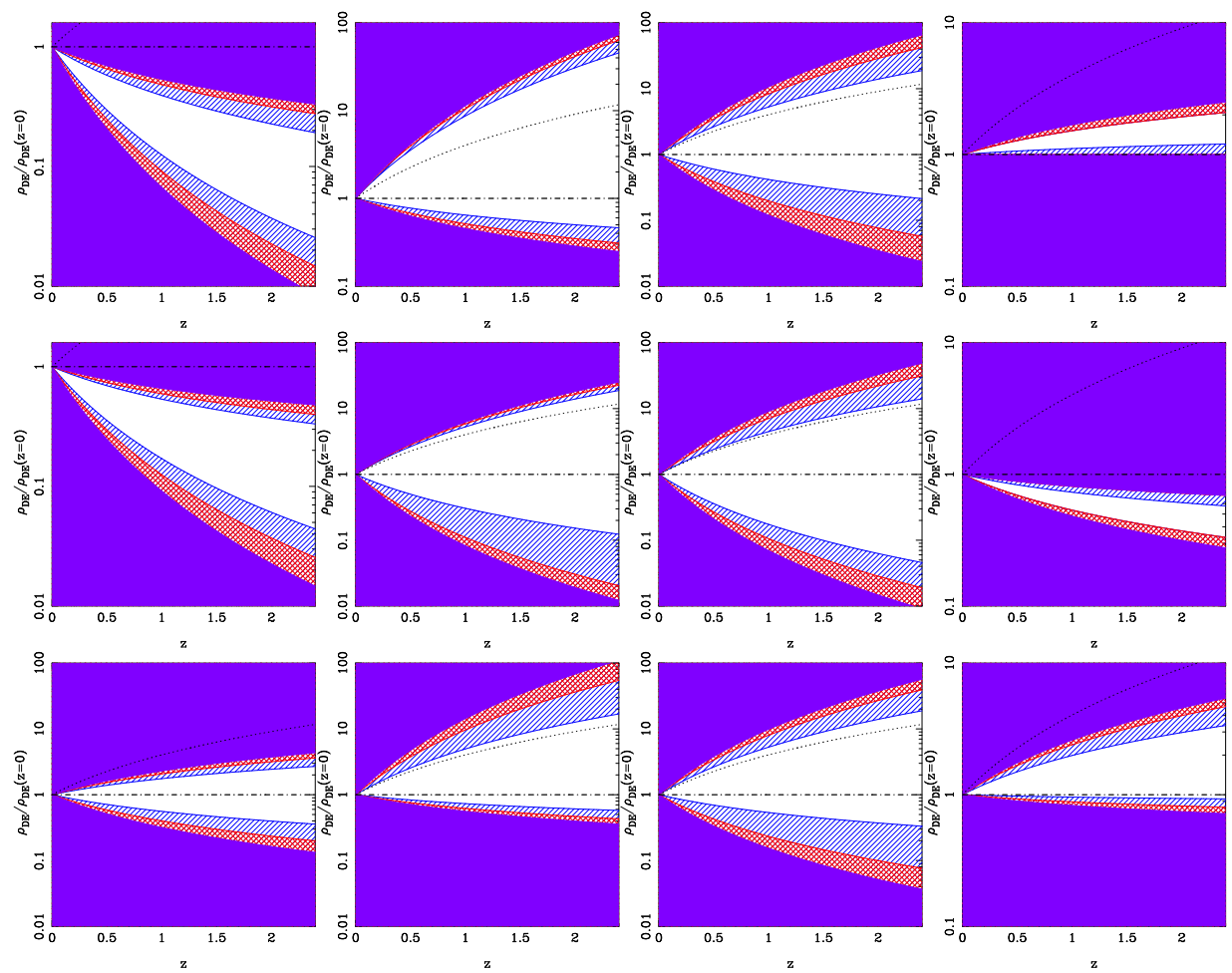

Figure 3. The plots represent the variation of dark energy density as a function of redshift allowed at 1,2 and $3-\sigma$ confidence levels. From left to right the ranges allowed by SNIa, BAO, H(z) and a combination of the datasets for the two different parameterizations with marginalization over $w^{\prime}$. The three rows correspond the models in 2

[53-61] in the range $0.07 \leq z \leq 2.3$.

We use the $\chi^{2}$ minimization technique to analyze the data and constrain cosmological parameters of interest in this work. For each data set, the observed quantity $X_{i, o b}$ at a certain redshift $z_{i}$, is compared with that obtained theoretically, $X_{i, t h}$, at the same redshift, for each class of dark energy models. The $\chi^{2}$ is defined as

$$
\chi^{2}=\sum_{i}\left[\frac{X_{i, o b}-X_{i, t h}}{\sigma_{i}}\right]^{2}
$$

The absolute magnitude of supernova is a nuisance parameter as far as dark energy parameters are concerned, and as the determination of absolute determination is degenerate with determination of value of Hubble constant, and the latter is also then a nuisance parameter. We consider the distance modulus given in [9] and for consistency with the earlier analyses we fix the value of $H_{0}$ to $70 \mathrm{~km} / \mathrm{s} / M p c$ (see also, [62]). Similarly, while analyzing the BAO observations, the $D_{v}(z)$ and $A(z)$ have been determined using $H_{0}=70 \mathrm{~km} / \mathrm{s} / M p c$. We are interested primarily in dark energy parameters, hence we marginalize over the present value of the Hubble parameter while analyzing $H(z)$ [53-61]. We consider the range $H_{0}=65-75 \mathrm{~km} / \mathrm{s} / \mathrm{Mpc}$ for this analysis and marginalize over this range as mentioned above. The priors are based on the results obtained using earlier, independent observations. For $w \mathrm{CDM}$ model, the priors used are $\Omega_{m}=0.01-0.6$, and $w=-4.0-0$. The priors for this analysis are listed in Table. 1. For models with a varying equation of state parameter, the present day value of dark energy equation of state $w_{0}$ is taken in the range -5.0 to 2.0 and $w^{\prime}(z=0)$ varies from -10.0 to 10.0. These priors are listed in Table. 2 . 

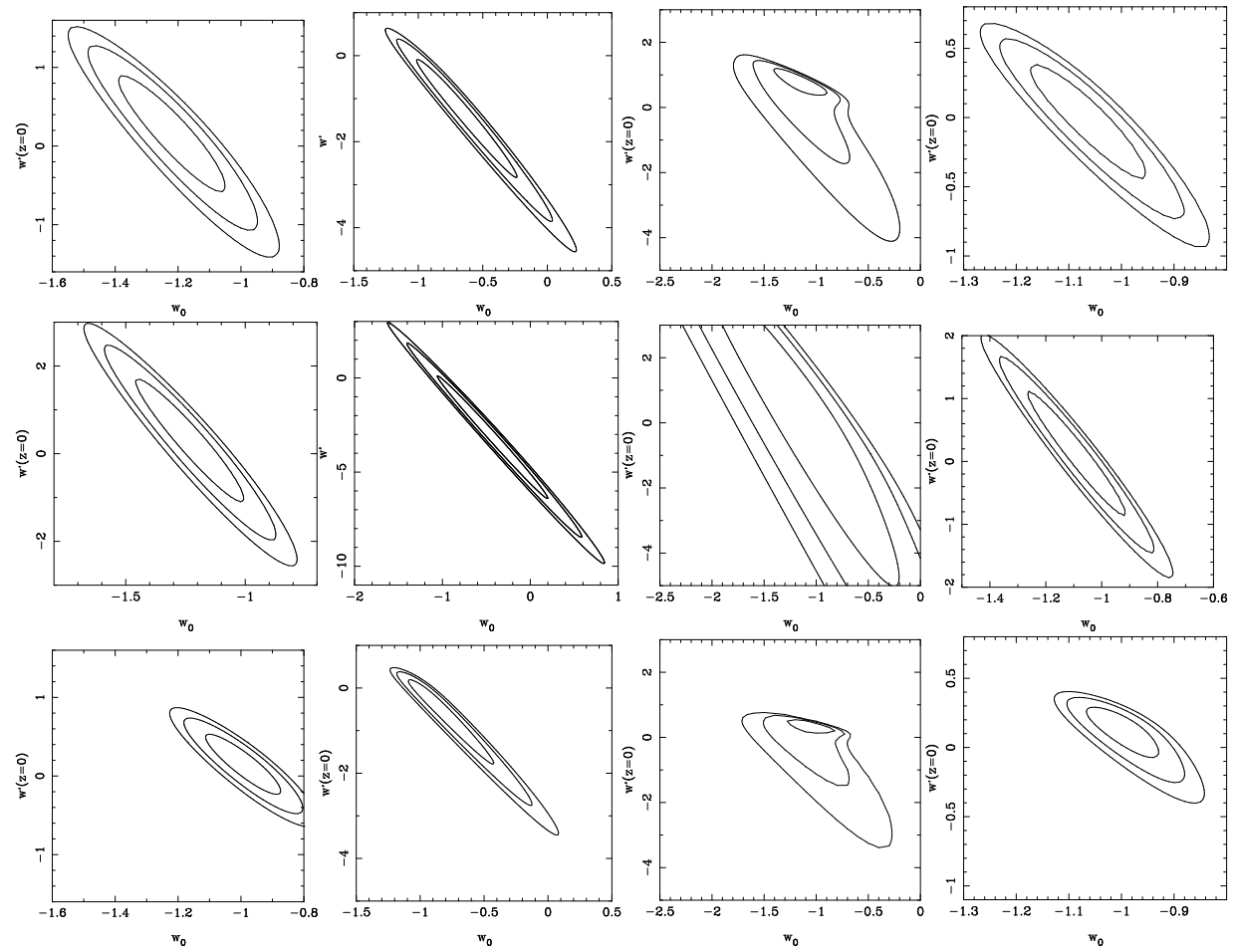

Figure 4. The plots represent the confidence contours for (from left to right) SNIa, BAO, H(z) and a combination of the datasets for the three different parameterizations with marginalization over $\Omega_{m}$. The contours in top row and the second row are for parameterizations $p=1$ and $p=2$ respectively and the last row corresponds to the logarithmic paramterization.

In Fig. 1, we show the constraints using these datasets for a cosmological model with constant equation of state parameter for dark energy ( $w \mathrm{CDM}$ model). This figure shows $1 \sigma, 2 \sigma$ and $3 \sigma$ contours corresponding to $67.3 \%, 95 \%$ and $99 \%$ confidence levels in the $\Omega_{m}-w$ plane. Going from left to right, the plots correspond to the confidence contours obtained by analyzing SNIa data, BAO data, $\mathrm{H}(\mathrm{z})$ data and their combination, respectively. The minimum value $\chi_{m}^{2}=562.29$ corresponds to the values $w=-1.04$ and $\Omega_{m}=0.29$ for SNIa data. In case of the BAO dataset, $\chi_{m}^{2}=0.96$ for $w=-1.2$ and $\Omega_{m}=0.27$. With $H(z)$ data, $\chi_{m}^{2}=16.27$ for $w=-1.12$ and $\Omega_{m}=0.28$. It is apparent from the figure that the $\mathrm{BAO}$ observations provide constraints which are complementary to those obtained from the other two observations and hence when combined, the allowed range of cosmological parameters is constrained very strongly. In this set of models with a constant equation of state parameter, the combined constraints are consistent with a cosmological constant. The confidence limits for these results are listed in Table 3.

In dark energy models where $w$ is a function of redshift, there are four parameters: $\Omega_{m}, w_{0}$, $w^{\prime}(z=0)$ and $H_{0}$. The priors for these parameters are listed in Table 2. In Figs. 2 and 3, we show confidence contours in $\Omega_{m}-w_{0}$ plane and $\rho_{D E} / \rho_{D E}(z=0)$ respectively with marginalization over $w^{\prime}$. For $\mathrm{H}(\mathrm{z})$ data we have marginalized over $H_{0}$ and in case of SNIa and BAO data, $H_{0}=70 \mathrm{~km} / \mathrm{s} / \mathrm{Mpc}$. The dotted line and dashed line (plotted here for reference) in the energy density plots corresponds to $\mathrm{w}=-1 / 3$ and $\mathrm{w}=-1$ respectively. In Figs. 4 and 5, we show confidence contours in $w^{\prime}-w_{0}$ plane and variation in dark energy density corresponding to the allowed range in $w_{0}$ and $w^{\prime}$ with marginalization over $\Omega_{m}$.

While the allowed ranges in $w_{0}$ and $w^{\prime}$ are different for these scenarios, the allowed ranges of 

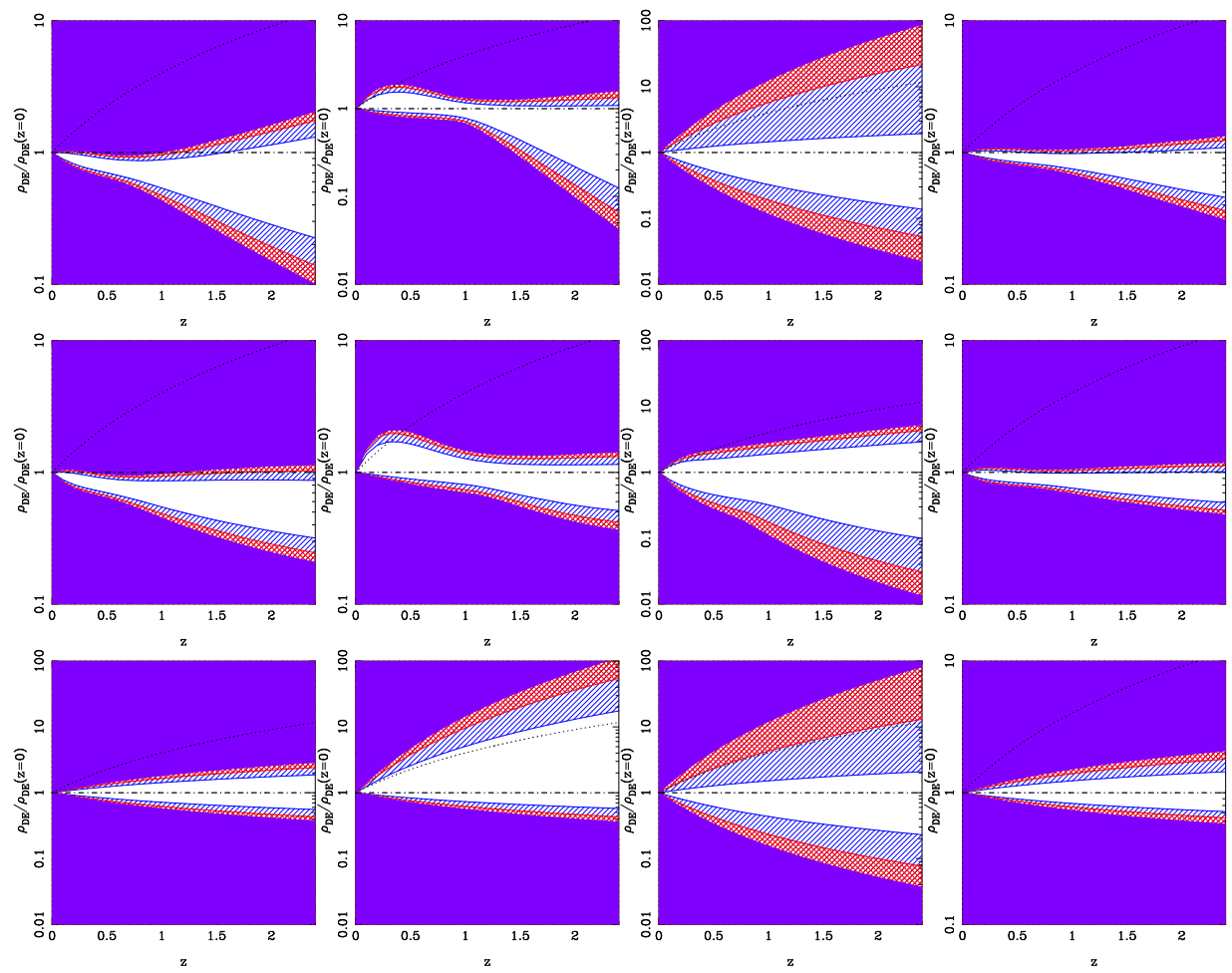

Figure 5. The plots show the allowed range of dark energy density by SNIa, BAO, H(z) and a combined datasets for the three different parameterizations with marginalization over $\Omega_{m}$.

dark energy density are comparable at lower redshifts $(\mathrm{z}<1)$. For higher $\operatorname{redshifts}(\mathrm{z}>1)$, the $p=2$ parameterization allows for a smaller range of allowed dark energy density. This is due to the fact that the asymptotic value at large redshifts approaches $w_{0}+w^{\prime}$ for $p=1$ parameterization and for $p=2$ the equation of state parameter reverts to the value $w_{0}$ A larger range is allowed in the case of the logarithmic parameterization as the equation of state parameter increases monotonically. Since dark energy is subdominant at redshifts larger than unity, this variation does not make a significant difference to the observational constraints.

The SNIa data shows a significant preference for 'phantom' models and rules out cosmological constant. The SNIa data prefers higher values of $\Omega_{m}$ for both the parameterizations. The other two datasets are at tension with the supernova data, the BAO data and the $H(z)$ data prefer 'quintessence' models and also allow a non-accelerating universe. The last two datasets are consistent with a cosmological model for the two different parameterizations considered here. These full range of all the free parameters used in the analysis are summarized in Table 4. In Fig. 6 we show the allowed range of dark energy density for this case. The allowed variation in dark energy density is larger to that obtained by marginalization over $\Omega_{m}$, however the combined range is similar in the two cases. While the individual observations allow a cosmological constant model, the preference for an equation of state with $w<-1$ by the supernova data dominates the combined results and disallow a cosmological constant.

\section{Summary and Conclusions}

In this paper, we have investigated constraints from current observations on dark energy equation of state parameter and its evolution at low redshifts. In order to do this analysis, we considered the 

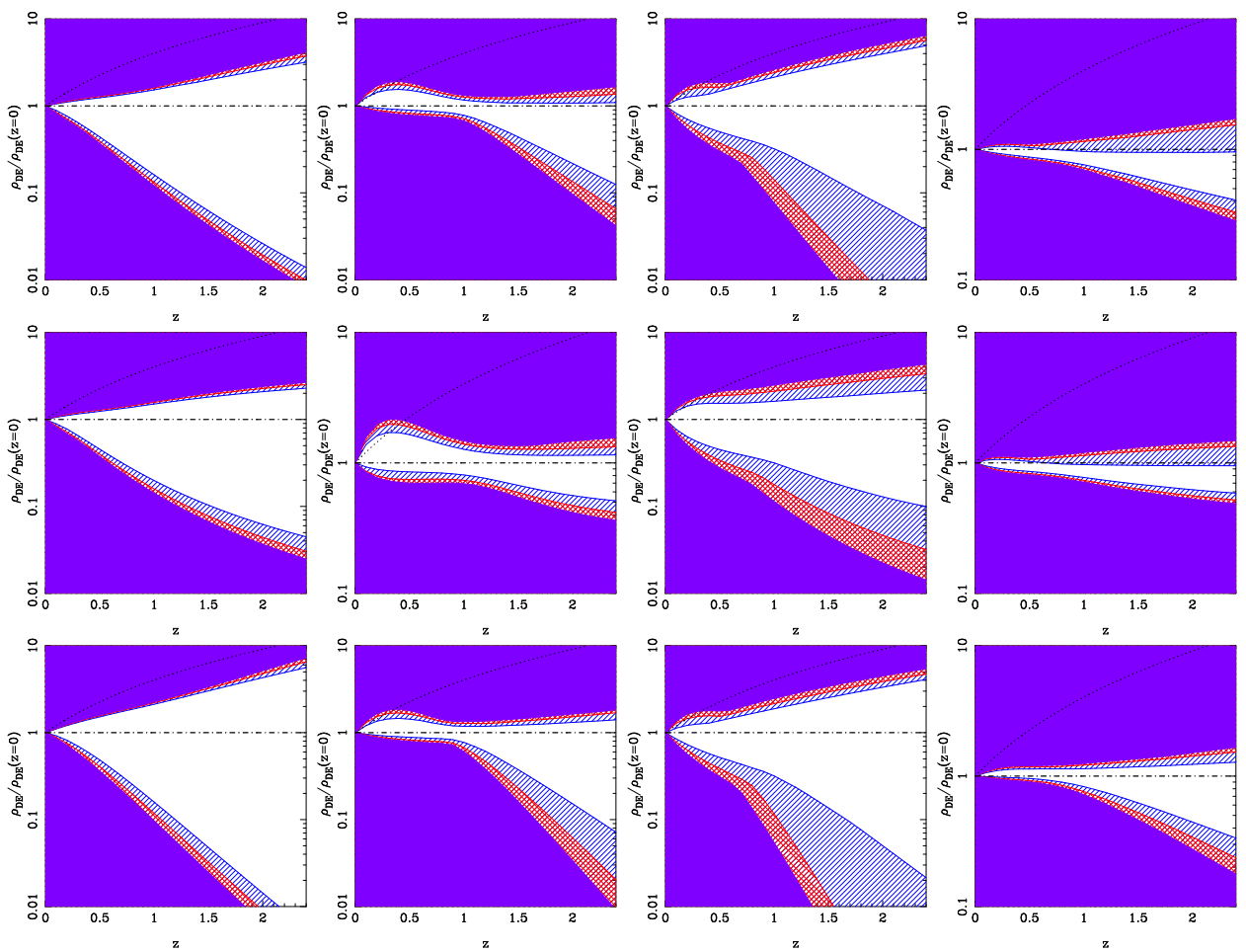

Figure 6. The plots show the allowed range of dark energy density by SNIa, BAO, H(z) and a combined datasets for the three different parameterizations with all parameters free.

Supernova Type Ia data, Baryon Acoustic Oscillation data and direct measurements of the Hubble parameter $\mathrm{H}(\mathrm{z})$ at different redshifts. We analyse the data separately and also combine the constraints obtained from these observations.

While the observations support the concordant cosmological constant model, the data does not rule out a constant dark energy which is not a cosmological constant and also allows some variation in the equation of state parameter. These datasets put comparable limits on evolution of dark energy density as a function of redshift and combining the datasets leads to significant reduction in the allowed range of dark energy density evolution. The constraints from the BAO data are complementary to those obtained by the SNIa data and therefore the combination is more effective in ruling out a large range of paramters.

We extend the analysis to models with a varying equation of state parameter and we consider three different functions of redshift. While the parameterizations considered here may be different in their nature, the allowed evolution of dark energy density is fairly model independent, especially if the observations are combined. For those models in which the equation of state parameter is a function of redshift, there is a tension between the SNIa data and other data sets considered in this paper, as SNIa dataset prefer phantom like models, with $w<-1$ over the quintessence $(w>-1)$.

The Planck best-fit base $\Lambda \mathrm{CDM}$ cosmology is in good agreement with results from BAO surveys, and with the recent Joint Light Curve Analysis (JLA) sample of SNIa. The Hubble parameter observations too, in combination with either of these observations, significantly reduce the allowed range. The consistency of combined observational constraints with a cosmological constant is primarily due to the $\mathrm{BAO}$ and $\mathrm{H}(\mathrm{z})$ measurement data. This is due to the large range of redshifts spanned by these datasets. More BAO and $H(z)$ observations at high and intermediate redshifts would further limit the number of viable cosmological models in addition to currently available data. 


\begin{tabular}{|c|c|c|c|}
\hline Data set & $3 \sigma$ confidence & $\chi_{m}^{2}$ & Best Fit Model \\
\hline \multicolumn{4}{|c|}{$p=1$} \\
\hline SNIa & $\begin{array}{l}-1.64 \leq w_{0} \leq-0.72 \\
-2.0 \leq w^{\prime}(z=0) \leq 1.26 \\
0.2 \leq \Omega_{m} \leq 0.45\end{array}$ & 562.25 & $\begin{array}{l}w_{0}=-1.0 \\
w^{\prime}(z=0)=0.2 \\
\Omega_{m}=0.25\end{array}$ \\
\hline $\mathrm{BAO}$ & $\begin{array}{l}-1.3 \leq w_{0} \leq 0.33 \\
-4.97 \leq w^{\prime}(z=0) \leq 0.77 \\
0.3 \leq \Omega_{m} \leq 0.31\end{array}$ & 2.13 & $\begin{array}{l}w_{0}=-0.67 \\
w^{\prime}(z=0)=-1.26 \\
\Omega_{m}=0.3\end{array}$ \\
\hline $\mathrm{H}(\mathrm{z})$ & $\begin{array}{l}-2.14 \leq w_{0} \leq 0.28 \\
-5.0 \leq w^{\prime}(z=0) \leq 1.8 \\
0.1 \leq \Omega_{m} \leq 0.37\end{array}$ & 20.77 & $\begin{array}{l}w_{0}=-1.16 \\
w^{\prime}(z=0)=0.8 \\
\Omega_{m}=0.2\end{array}$ \\
\hline $\mathrm{SNIa}+\mathrm{BAO}+\mathrm{H}(\mathrm{z})$ & $\begin{array}{l}-1.2 \leq w_{0} \leq-0.74 \\
-1.32 \leq w^{\prime}(z=0) \leq 0.56 \\
0.25 \leq \Omega_{m} \leq 0.3\end{array}$ & 585.67 & $\begin{array}{l}w_{0}=-1.0 \\
w^{\prime}(z=0)=-0.26 \\
\Omega_{m}=0.3\end{array}$ \\
\hline \multicolumn{4}{|c|}{$p=2$} \\
\hline SNIa & $\begin{array}{l}-1.62 \leq w_{0} \leq-0.62 \\
-3.0 \leq w^{\prime}(z=0) \leq 2.56 \\
0.2 \leq \Omega_{m} \leq 0.45\end{array}$ & 562.28 & $\begin{array}{l}w_{0}=-1.06 \\
w^{\prime}(z=0)=-0.06 \\
\Omega_{m}=0.3\end{array}$ \\
\hline $\mathrm{BAO}$ & $\begin{array}{l}-1.68 \leq w_{0} \leq 0.99 \\
-10.0 \leq w^{\prime}(z=0) \leq 2.99 \\
0.25 \leq \Omega_{m} \leq 0.3\end{array}$ & 2.79 & $\begin{array}{l}w_{0}=-0.44 \\
w^{\prime}(z=0)=-3.06 \\
\Omega_{m}=0.3\end{array}$ \\
\hline$\overline{\mathrm{H}(\mathrm{z})}$ & $\begin{array}{l}-2.36 \leq w_{0} \leq 0.24 \\
-5.8 \leq w^{\prime}(z=0) \leq 3.4 \\
0.18 \leq \Omega_{m} \leq 0.37\end{array}$ & 21.11 & $\begin{array}{l}w_{0}=-1.16 \\
w^{\prime}(z=0)=0.3 \\
\Omega_{m}=0.28\end{array}$ \\
\hline $\mathrm{SNIa}+\mathrm{BAO}+\mathrm{H}(\mathrm{z})$ & $\begin{array}{l}-1.36 \leq w_{0} \leq-0.64 \\
-2.52 \leq w^{\prime}(z=0) \leq 1.62 \\
0.25 \leq \Omega_{m} \leq 0.3\end{array}$ & 586.46 & $\begin{array}{l}w_{0}=-1.0 \\
w^{\prime}(z=0)=-0.38 \\
\Omega_{m}=0.3\end{array}$ \\
\hline \multicolumn{4}{|c|}{ Logarithmic } \\
\hline SNIa & $\begin{array}{l}-1.44 \leq w_{0} \leq-0.58 \\
-2.0 \leq w^{\prime}(z=0) \leq 0.68 \\
0.1 \leq \Omega_{m} \leq 0.49\end{array}$ & 563.02 & $\begin{array}{l}w_{0}=-0.94 \\
w^{\prime}(z=0)=-1.04 \\
\Omega_{m}=0.37\end{array}$ \\
\hline $\mathrm{BAO}$ & $\begin{array}{l}-1.26 \leq w_{0} \leq 0.2 \\
-3.8 \leq w^{\prime}(z=0) \leq 0.5 \\
0.26 \leq \Omega_{m} \leq 0.32\end{array}$ & 1.29 & $\begin{array}{l}w_{0}=-0.84 \\
w^{\prime}(z=0)=-0.5 \\
\Omega_{m}=0.29\end{array}$ \\
\hline $\mathrm{H}(\mathrm{z})$ & $\begin{array}{l}-2.0 \leq w_{0} \leq 0.2 \\
-5.0 \leq w^{\prime}(z=0) \leq 0.9 \\
0.1 \leq \Omega_{m} \leq 0.37\end{array}$ & 20.91 & $\begin{array}{l}w_{0}=-1.1 \\
w^{\prime}(z=0)=0.3 \\
\Omega_{m}=0.24\end{array}$ \\
\hline SNIa+BAO+H(z) & $\begin{array}{l}-1.09 \leq w_{0} \leq-0.66 \\
-1.21 \leq w^{\prime}(z=0) \leq 0.25 \\
0.26 \leq \Omega_{m} \leq 0.32\end{array}$ & 587.18 & $\begin{array}{l}w_{0}=-0.91 \\
w^{\prime}(z=0)=-0.29 \\
\Omega_{m}=0.29\end{array}$ \\
\hline
\end{tabular}

Table 4. This table shows the $3 \sigma$ confidence limit for various data sets for the different varying dark energy models for $H_{0}=70 \mathrm{~km} / \mathrm{s} / \mathrm{Mpc}$ for BAO and SNIa and for $\mathrm{H}(\mathrm{z})$ data, marginalized over $H_{0}$. For these results, we have kept $\Omega_{m}$ a free parameter.

\section{Acknowledgments}

HKJ and AT thank Department of Science and Technology (DST), Delhi for funding via project SR/FTP/PS-127/2012. The numerical work presented in this paper was done on high computing 
facility at IISER Mohali.

\section{References}

[1] Perlmutter, S., Gabi, S., Goldhaber, G., et al., Measurements of the Cosmological Parameters $\Omega$ and $\Lambda$ from the First Seven Supernovae at $z \geq 0.35$, Astrophys. J.483 (1997) 565.

[2] Perlmutter, S., Aldering, G., Goldhaber, G., et al., Measurements of $\Omega$ and $\Lambda$ from 42 High-Redshift Supernovae, Astrophys. J.517 (1999) 565.

[3] Riess, A. G., Filippenko, A. V., Challis, P., et al., Observational Evidence from Supernovae for an Accelerating Universe and a Cosmological Constant, Astrophys. J.116 (1998) 1009.

[4] Astier, P., Guy, J., Regnault, N., et al., The Supernova Legacy Survey: measurement of $\Omega_{M}, \Omega_{\Lambda}$ and $w$ from the first year data set, Astron. Astrophys.447 (2006) 31.

[5] Garnavich, P. M., Jha, S., Challis, P., et al., Supernova Limits on the Cosmic Equation of State, Astrophys. J.509 (1998) 74.

[6] Tonry, J. L. et al., Cosmological Results from High-z Supernovae, Astrophys. J.594 (2003) 1.

[7] Barris, B. J. et al.,Twenty-Three High-Redshift Supernovae from the Institute for Astronomy Deep Survey: Doubling the Supernova Sample at z > 0.7, Astrophys. J.602 (2004) 571.

[8] Goobar, A. et al., The Acceleration of the Universe: Measurements of Cosmological Parameters from Type la Supernovae, Physica Scripta Volume T 85 (2000) 47.

[9] González-Gaitán, S., Conley, A., Bianco, F. B., et al., The Rise Time of Normal and Subluminous Type Ia Supernovae, Astrophys. J.745 (2012) 44.

[10] Suzuki, N., Rubin, D., Lidman, C., et al., The Hubble Space Telescope Cluster Supernova Survey. V. Improving the Dark-energy Constraints above $z>1$ and Building an Early-type-hosted Supernova Sample, Astrophys. J.746 (2012) 85.

[11] Caldwell, R. R., Dark energy, Phys. World 17 (2004) 37.

[12] Padmanabhan, T., Cosmological constant-the weight of the vacuum, Phys. Rept.380 (2003) 235.

[13] Coble, K., Dodelson, S., and Frieman, J. A., Dynamical $\Lambda$ models of structure formation, Phys. Rev. D55 (1997) 1851.

[14] Peebles, P. J., and Ratra, B., The cosmological constant and dark energy, Reviews of Modern Physics 75 (2003) 559.

[15] Caldwell, R. R. and Dave, R. and Steinhardt, P. J., Cosmological Imprint of an Energy Component with General Equation of State, Physical Review Letters 80 (1998) 1582.

[16] Weinberg S., The cosmological constant problem, Reviews of Modern Physics 61 (1989) 1.

[17] Carroll, S. M. and Press, W. H. and Turner, E. L., The cosmological constant, Annu. Rev. Astron. and Astrophys.30 (1992) 499.

[18] Sahni V., Starobinsky A. A., The Case for a Positive Cosmological $\Lambda$-Term, International Journal of Modern Physics D 9 (2000) 373.

[19] Albrecht, A., Bernstein, G., Cahn, R., et al., Report of the Dark Energy Task Force, (2006) arXiv:astro-ph/0609591.

[20] Frieman, J. A., Turner, M. S., and Huterer, D., Dark Energy and the Accelerating Universe, Annu. Rev. Astron. and Astrophys.46 (2008) 385.

[21] Linder, E. V., Mapping the cosmological expansion, Reports on Progress in Physics 71 (2008) 056901.

[22] Ellis, J., Dark matter and dark energy: summary and future directions, Philosophical Transactions of the Royal Society of London Series A 361 (2003) 2607. 
[23] Sami, M., A primer on problems and prospects of dark energy, (2009) arXiv:0904.3445.

[24] Ratra, B. and Peebles, P. J. E., Cosmological consequences of a rolling homogeneous scalar field, Phys. Rev. D37 (1988) 3406.

[25] Caldwell, R. R., and Kamionkowski, M., The Physics of Cosmic Acceleration, Annual Review of Nuclear and Particle Science 59 (2009) 397.

[26] Silvestri, A., and Trodden, M., Approaches to understanding cosmic acceleration, Reports on Progress in Physics 72 (2009) 096901.

[27] Chevallier, M. and Polarski, D., Accelerating Universes with Scaling Dark Matter, International Journal of Modern Physics D 10 (2001)213.

[28] Linder, E. V., Exploring the Expansion History of the Universe, Physical Review Letters 90 (2003) 091301.

[29] Jassal, H. K., Bagla, J. S., and Padmanabhan, T., Observational constraints on low redshift evolution of dark energy: How consistent are different observations?, Phys. Rev. D72 (2005) 103503.

[30] Lei Feng and Tan Lu, A New Equation of State for Dark Energy Model, Journal of Cosmology and Astroparticle Physics 11 (2011) 34.

[31] Efstathiou, G., Constraining the equation of state of the Universe from distant Type Ia supernovae and cosmic microwave background anisotropies, Mon. Not. Roy. Astron. Soc.310 (1999) 842.

[32] Lee, S., Constraints on the dark energy equation of state from the separation of CMB peaks and the evolution of $\alpha$, Phys. Rev. D71 (2005) 123528.

[33] Li, M., A model of holographic dark energy, Physics Letters B 603 (2004) 1.

[34] Hannestad, S. and Mörtsell, E., Cosmological constraints on the dark energy equation of state and its evolution, JCAP 09 (2004) 01.

[35] Wang, Y., Kostov, V. and Freese, K. and Frieman, J. A. and Gondolo, P., Probing the evolution of the dark energy density with future supernova surveys, JCAP 12 (2004).

[36] Wang, Y., and Tegmark, M., New Dark Energy Constraints from Supernovae, Microwave Background, and Galaxy Clustering, Physical Review Letters 92 (2004) 241302.

[37] Bassett, B. A., Corasaniti, P. S., and Kunz, M., The Essence of Quintessence and the Cost of Compression, Astrophys. J. Lett.617 (2004) L1.

[38] Huterer, D. and Turner, M. S., Prospects for probing the dark energy via supernova distance measurements, Phys. Rev. D60 (1999) 081301.

[39] Huterer, D. and Turner, M. S., Probing dark energy: Methods and strategies, Phys. Rev. D64 (2001) 123527.

[40] Weller, J and Albrecht, A., Opportunities for Future Supernova Studies of Cosmic Acceleration, Physical Review Letters 86 (2001) 1939.

[41] Pantazis, G., Nesseris, S., and Perivolaropoulos, L., Comparison of thawing and freezing dark energy parametrizations, Phys. Rev. D93 (2016) 103503.

[42] Rani, N., Jain, D., Mahajan, S.,Mukherjee A. and Pires, N., Transition Redshift: New constraints from parametric and nonparametric methods, JCAP 1512 (2015) 45.

[43] Copeland, E. J. and Sami, M. and Tsujikawa, S., Dynamics of Dark Energy, International Journal of Modern Physics D 15 (2006) 1753.

[44] Bamba, K., Capozziello, S., Nojiri, S., and Odintsov, S. D., Dark energy cosmology: the equivalent description via different theoretical models and cosmography tests, Astrophys.Space Sci. 342 (2012) 155. 
[45] Capozziello, S., Cardone, V. F., Elizalde, E., Nojiri, S., and Odintsov, S. D., Observational constraints on dark energy with generalized equations of state, Phys. Rev. D73 (2006) 043512.

[46] Seo, H.-J., and Eisenstein, D. J., Probing Dark Energy with Baryonic Acoustic Oscillations from Future Large Galaxy Redshift Surveys, Astrophys. J.598 (2003) 720.

[47] Percival, W. J., Nichol, R. C., Eisenstein, D. J., et al., The Shape of the Sloan Digital Sky Survey Data Release 5 Galaxy Power Spectrum, Astrophys. J.657 (2007) 645.

[48] Busca, N. G. et al, Baryon acoustic oscillations in the Ly $\alpha$ forest of BOSS quasars, Astron. Astrophys.552 (2013) A96.

[49] Blake, C., Brough, S., Colless, M., et al., The WiggleZ Dark Energy Survey: joint measurements of the expansion and growth history at $z<1$, Mon. Not. Roy. Astron. Soc.425 (2012) 405.

[50] Anderson, L., Aubourg, Bailey, S., et al., The clustering of galaxies in the SDSS-III Baryon Oscillation Spectroscopic Survey: baryon acoustic oscillations in the Data Releases 10 and 11 Galaxy samples, Mon. Not. Roy. Astron. Soc.441 (2014) 24.

[51] Veropalumbo, A., Marulli, F., Moscardini, L., Moresco, M., and Cimatti, A., An improved measurement of baryon acoustic oscillations from the correlation function of galaxy clusters at $z \sim 0.3$, Mon. Not. Roy. Astron. Soc.442 (2014) 3275.

[52] Delubac, T., et al. Baryon acoustic oscillations in the Ly $\alpha$ forest of BOSS DR11 quasars, Astron. Astrophys.574 (2015) A59.

[53] Samushia, L., and Ratra, B., Cosmological Constraints from Hubble Parameter versus Redshift Data, Astrophys. J. Lett.650 (2006) L5.

[54] Simon, J., Verde, L., and Jimenez, R., Constraints on the redshift dependence of the dark energy potential, Phys. Rev. D71 (2005) 123001.

[55] Stern, D. and Jimenez, R. and Verde, L. and Kamionkowski, M. and Stanford, S. A., Cosmic chronometers: constraining the equation of state of dark energy. I: H(z) measurements, JCAP 2 (2010) 008 .

[56] Farooq, O. and Ratra, B., Hubble Parameter Measurement Constraints on the Cosmological Deceleration-Acceleration Transition Redshift, The Astrophysical Journal Letters 766 (2013) L7.

[57] Farooq, O. and Mania, D. and Ratra, B., Hubble Parameter Measurement Constraints on Dark Energy, Astrophys. J.764 (2013) 138.

[58] Moresco, M. et al, Improved constraints on the expansion rate of the Universe up to $z^{\sim} 1.1$ from the spectroscopic evolution of cosmic chronometers, JCAP 8 (2012) 006.

[59] Zhang, C. and Zhang, H. and Yuan, S. and Liu, S. and Zhang, T.-J. and Sun, Y.-C., Four new observational $H(z)$ data from luminous red galaxies in the Sloan Digital Sky Survey data release seven, Research in Astronomy and Astrophysics 14 (2014) 1221.

[60] Chuang, C.-H. and Wang, Y., Modelling the anisotropic two-point galaxy correlation function on small scales and single-probe measurements of $H(z), D_{A}(z)$ and $f(z) \sigma_{8}(z)$ from the Sloan Digital Sky Survey DR7 luminous red galaxies, Mon. Not. Roy. Astron. Soc.435 (2013) 255.

[61] Chen, Y. and Ratra, B., Hubble parameter data constraints on dark energy, Physics Letters B 703 (2011) 406.

[62] Lukovic, V., Rocco D’Agostinoa and Vittorio, N, Is there a concordance value for H0?, arXIv: 1607.05677.

[63] Wei, J.-J., Wu, X.-F., and Melia, F., The Gamma-Ray Burst Hubble Diagram and Its Implications for Cosmology, Astrophys. J.772 (2013) 43.

[64] Li, X., Hjorth, J., and Wojtak, R., Cosmological Parameters from Supernovae Associated with Gamma-Ray Bursts, Astrophys. J. Lett.796 (2014) L4. 
[65] Wang, F. Y., and Dai, Z. G., Estimating the uncorrelated dark energy evolution in the Planck era, Phys. Rev. D89 (2014) 023004.

[66] Wang, F. Y., Dai, Z. G., and Liang, E. W., Gamma-ray burst cosmology, New Atron. Rev.67 (2015) 1.

[67] Wang, F.-Y., Qi, S., and Dai, Z.-G., The updated luminosity correlations of gamma-ray bursts and cosmological implications, Mon. Not. Roy. Astron. Soc.415 (2011) 3423.

[68] Wei, J.-J., Wu, X.-F., and Melia, F., Cosmological tests using the angular size of galaxy clusters, Mon. Not. Roy. Astron. Soc.447 (2015) 479.

[69] Kovetz, E. D., Raccanelli, A., and Rahman, M., Cosmological Constraints with Clustering-Based Redshifts, (2016) arXiv:1606.07434.

[70] Planck Collaboration, Ade, P. A. R., Aghanim, N., et al., Planck 2015 results. XIII. Cosmological parameters, Astron. Astrophys.594 (2016) A13.

[71] Planck Collaboration, Ade, P. A. R., Aghanim, N., et al., Planck 2013 results. XVI. Cosmological parameters, Astron. Astrophys.571 (2014) A16.

[72] Farooq, O., Ranjeet Madiyar, F., Crandall, S., and Ratra, B., Hubble Parameter Measurement Constraints on the Redshift of the Deceleration-Acceleration Transition, Dynamical Dark Energy, and Space Curvature, Astrophys. J.835 (2017) 26.

[73] Spergel, D. N., Bean, R., Dore, O., et al., Three-Year Wilkinson Microwave Anisotropy Probe (WMAP) Observations: Implications for Cosmology, Astrophys. J. Suppl.170 (2007) 377. 